\title{
ABBREVIATIONS AND PRIMARY SOURCES
}

In addition to explaining abbreviations used in this book, the following list includes, for students wishing to consult the original Greek sources, the Greek texts used or cited in the book. Greek texts of the authors listed are also available in the Loeb Classical Library series published by Harvard University Press. The Loeb editions provide the Greek text and an English translation on facing pages.

Aristophanes

Aristophanis Comoediae. Edited by F. W. Hall and W. M. Geldart. Oxford, I9o6.

[Aristotle] Constitution of the Athenians

Aristotelis Atheniensium Respublica. Edited by F. G. Kenyon. Oxford, I920.

Aristotle Rhetoric

Aristotelis Ars rhetorica. Edited by W. D. Ross. Oxford, I959. 
FgrHist

Die Fragmente der griechischen Historiker. Edited by F. Jacoby. Berlin and Leiden, I923-.

Herodotus

Herodoti Historiae. Edited by C. Hude. Oxford, I927. $I G \mathrm{I}^{3}$

Inscriptiones Graecae. Vol. I, Inscriptiones Atticae anno Euclidis anteriores. $3 \mathrm{rd}$ ed. Fasc. I edited by D. Lewis; fasc. 2 edited by D. Lewis and L. Jeffery. Berlin and New York, I98I and I994.

$I G \mathrm{II}^{2}$

Inscriptiones Graecae Euclidis anno posteriores. Vols. 2-3. and ed. Edited by J. Kirchner. Berlin, I9I3-r93I.

Isocrates

Isocrates. 3 vols. Edited and translated by G. Norlin and L. van Hook. Loeb Classical Library. Cambridge, Mass., I929-I945.

Lysias

Lysiae Orationes. Edited by C. Hude. Oxford, I9I2. $P C G$

Poetae comici Graeci. Edited by R. Kassel and C. Austin. Berlin and New York, 1983-.

Plato

Platonis Opera. Edited by J. Burnet. Oxford, I90I-I907. Plutarch

Plutarchi Vitae parallelae. Vol. I.2. Edited by K. Ziegler. Leipzig, I964.

Sophocles

Sophoclis Fabulae. Edited by H. Lloyd-Jones and N.G. Wilson. Oxford, I992. 
Abbreviations and Primary Sources / $\mathrm{xix}$

Thucydides

Thucydidis Historiae. Edited by H. S. Jones. Oxford, I942. Xenophon

Xenophontis Opera omnia. Edited by E. C. Marchant. Oxford, I9I3. 
This page intentionally left blank 